\title{
Multivariate Varying Coefficient Models for DTI Tract Statistics ${ }^{\star}$
}

\author{
Hongtu Zhu, Martin Styner, Yimei Li, Linglong Kong, Yundi Shi, Weili Lin, \\ Christopher Coe, and John H. Gilmore
}

Department of Biostatistics, Radiology, Psychiatry and Computer Science, and Biomedical Research Imaging Center, University of North Carolina at Chapel Hill

\begin{abstract}
Diffusion tensor imaging (DTI) is important for characterizing the structure of white matter fiber bundles as well as detailed tissue properties along these fiber bundles in vivo. There has been extensive interest in the analysis of diffusion properties measured along fiber tracts as a function of age, diagnostic status, and gender, while controlling for other clinical variables. However, the existing methods have several limitations including the independent analysis of diffusion properties, a lack of method for accounting for multiple covariates, and a lack of formal statistical inference, such as estimation theory and hypothesis testing. This paper presents a statistical framework, called VCMTS, to specifically address these limitations. The VCMTS framework consists of four integrated components: a varying coefficient model for characterizing the association between fiber bundle diffusion properties and a set of covariates, the local polynomial kernel method for estimating smoothed multiple diffusion properties along individual fiber bundles, global and local test statistics for testing hypotheses of interest along fiber tracts, and a resampling method for approximating the $p$-value of the global test statistic. The proposed methodology is applied to characterizing the development of four diffusion properties along the splenium and genu of the corpus callosum tract in a study of neurodevelopment in healthy rhesus monkeys. Significant time effects on the four diffusion properties were found.
\end{abstract}

\section{Introduction}

In the existing literature, there are three major approaches to the group analysis of diffusion imaging data including region-of-interest (ROI) analysis, voxelwise analysis, and fiber tract based analysis [1, 2], 3]. The region-of-interest (ROI) method primarily computes averages diffusion properties in some manually drawn ROIs, generates various summary statistics per ROI, and then carries

\footnotetext{
* This work was supported in part by NSF grant BCS-08-26844 and NIH grants UL1RR025747-01, MH086633, P01CA142538-01 and AG033387 to Dr. Zhu, NIH grants MH064065, HD053000, and MH070890 to Dr. Gilmore, NIH grants R01NS055754 and R01EB5-34816 to Dr. Lin, Lilly Research Laboratories, the UNC NDRC HD 03110, Eli Lilly grant F1D-MC-X252, and NIH Roadmap Grant U54 EB005149-01, NAMIC to Dr. Styner.
} 
out statistical analysis on these summary statistics [3]. ROI analysis suffers from identifying meaningful ROIs, particularly the long curved structures common in fiber tracts, the instability of statistical results obtained from ROI analysis, and the partial volume effect in relative large ROIs.

Compared with the other two methods, voxel-wise analysis has been widely used in neuroimaging studies [4. The voxel-wise analysis involves two sequential steps. The first step is to fit a statistical model to diffusion properties at each voxel and generating a parametric map of test statistics (or $p$-values). The second step includes a correction to the multiple comparisons across the many voxels of imaging volume [5]. However, the voxel-wise analysis suffers from the issues of misalignment and arbitrary smoothing extent [6], 7]. As pointed out in [7, the final statistical results of voxel-wise analysis can strongly depend on the amount of smoothing applied to the diffusion tensor imaging data.

The third method is to develop fiber tract based analysis of diffusion properties, such as eigenvalues and fractional anisotropy (FA) values [1, 2, 8, , 9]. In [1], a tract-based spatial statistics framework is proposed to carry out a pointwise analysis along the white matter skeleton. In [8], a model-based framework is developed for the analysis of diffusion properties on the medial manifolds of fiber tracts followed by testing pointwise hypotheses on the medial manifolds. In [9], a functional principal component analysis (PCA) is used to compare a univariate diffusion property, such as fractional anisotropy, across two (or more) populations for a single hypothesis test per tract [9]. Furthermore, in [10, a constrained PCA method is proposed to fit age-related changes white matter diffusion of fiber tracts. The functional and constrained PCA methods suffer from the issues of the independent analysis of diffusion properties, a lack of method for accounting for multiple covariates, and a lack of formal statistical inference, such as estimation theory and hypothesis testing. In 11, a functional regression framework, called FRATS, is proposed for the analysis of multiple diffusion properties along fiber bundle as functions and their association with a set of covariates of interest in real applications.

The goal of this paper is to develop a multivariate varying coefficient model framework, called VCMTS, to completely address the issues of the functional and constrained PCA. Compared with the existing literature including [10, 11], and [9], we have made several novel contributions. We develop a multivariate varying coefficient model to statistically characterize the association between multiple fiber bundle diffusion properties and a set of covariates of interest. We use the local polynomial kernel method to regularize multiple diffusion properties along individual fiber bundles. We propose both local and global test statistics for testing hypothesis of interest along and on fiber tracts. We approximate the $p$-value of the global test statistic using a resampling method.

\section{Method}

A schematic overview of VCMTS is given in Figure 1. We have proven that each component of VCMTS is statistically sound under some mild conditions and the 


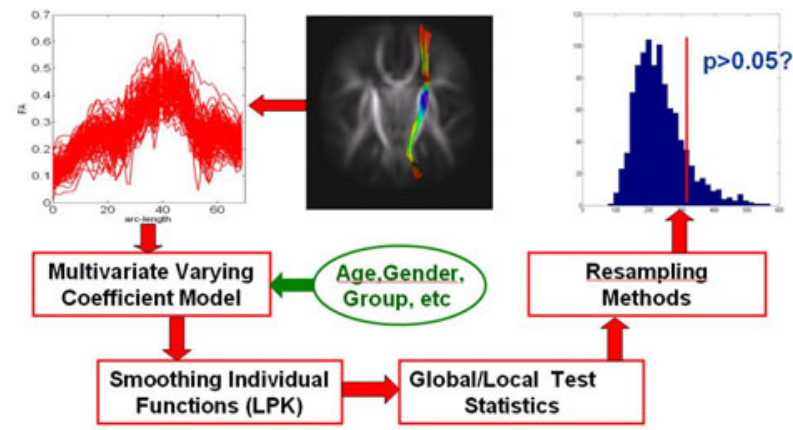

Fig. 1. A schematic overview of VCMTS, in which the right internal capsule tract is used as an illustration

detailed proof can be found in [12. The associated software for implementing VCMTS will be available in https://bios.unc.edu/ hzhu/ and disseminated to imaging researchers through http://www.nitrc.org/. We describe each of these components in detail below.

\subsection{Multivariate Varying Coefficient Model}

We develop a multivariate varying coefficient model to characterize the relationship between multiple diffusion properties along fiber tracts and a set of covariates of interest, such as age, group status, and gender. For the $i$-th subject, we consider an $m \times 1$ vector of diffusion properties, denoted by $\mathbf{y}_{i}\left(s_{j}\right)=$ $\left(y_{i, 1}\left(s_{j}\right), \cdots, y_{i, m}\left(s_{j}\right)\right)^{T}$, and its associated arc length $s_{j}$ for the $j$-th location grid point on the fiber bundle for $j=1, \cdots, n_{G}$ and $i=1, \cdots, n$, where $n_{G}$ and $n$ denote the numbers of grid points and subjects, respectively. We assume that

$$
y_{i, k}(s)=\mathbf{x}_{i}^{T} B_{k}(s)+\eta_{i, k}(s)+\epsilon_{i, k}(s),
$$

where $B_{k}(s)=\left(b_{k 1}(s), \cdots, b_{k p}(s)\right)^{T}$ is a $p \times 1$ vector of functions of $s, \mathbf{x}_{i}$ is a $p \times 1$ vector of covariates of interest, $\epsilon_{i, k}(s)$ are measurement errors and $\eta_{i, k}(s)$ characterize individual curve variations from $\mathbf{x}_{i}^{T} B_{k}(s)$. The varying coefficient matrix $B(s)=\left[B_{1}(s), \cdots, B_{m}(s)\right]$ characterizes the association between fiber bundle diffusion properties and the covariates of interest $\mathbf{x}_{i}$. Model (1) is a multivariate varying coefficient model [13]. Let $\mathrm{SP}(\mu, \Sigma)$ denote a stochastic process vector with mean function $\mu(t)$ and covariance function $\Sigma(s, t)$. We assume that $\boldsymbol{\epsilon}_{i}(s)=\left(\epsilon_{i, 1}(s), \cdots, \epsilon_{i, m}(s)\right)^{T}$ and $\boldsymbol{\eta}_{i}(s)=\left(\eta_{i, 1}(s), \cdots, \eta_{i, m}(s)\right)^{T}$ are independent, and $\boldsymbol{\eta}_{i}(s)$ and $\boldsymbol{\epsilon}_{i}(s)$ are independent and identical copies of $\operatorname{SP}\left(\mathbf{0}, \Sigma_{\eta}\right)$ and $\operatorname{SP}\left(\mathbf{0}, \Sigma_{\epsilon}\right)$, respectively. Moreover, $\epsilon_{i}(s)$ and $\epsilon_{i}\left(s^{\prime}\right)$ are assumed to be independent and thus $\Sigma_{\epsilon}(s, t)$ takes the form of $\Sigma_{\epsilon}(s, s) \mathbf{1}(s=t)$, where $\mathbf{1}(\cdot)$ is an indicator function. Finally, the covariance structure of $\mathbf{y}_{i}(s)$, denoted by $\Sigma_{y}(s, t)$, takes the form of $\Sigma_{y}(s, t)=\operatorname{Cov}\left(\mathbf{y}_{i}(s), \mathbf{y}_{i}(t)\right)=\Sigma_{\eta}(s, t)+\Sigma_{\epsilon}(s, s) \mathbf{1}(s=t)$.

As an illustration, in our clinical study on early rhesus monkey brain development, we are interested in studying the evolution of the three eigenvalues $\lambda_{i}$ 
of diffusion tensor $\left(\lambda_{1} \geq \lambda_{2} \geq \lambda_{3}\right)$ along two selected fiber tracts in 24 healthy rhesus monkeys (Fig. 2 (a)-(c)). See clinical data for details. We consider a model of $\lambda_{1}$ and $\lambda_{(2,3)}=\left(\lambda_{2}+\lambda_{3}\right) / 2$ along a specific tract as follows:

$$
\begin{aligned}
& \lambda_{i, 1}(s)=\beta_{11}(s)+\beta_{12}(s) \times \mathrm{g}_{i}+\beta_{13}(s) \times \operatorname{age}_{i}+\beta_{14}(s) \times \operatorname{age}_{i}^{2}+\eta_{i 1}(s), \\
& \lambda_{i,(2,3)}(s)=\beta_{21}(s)+\beta_{22}(s) \times \mathrm{g}_{i}+\beta_{23}(s) \times \operatorname{age}_{i}+\beta_{24}(s) \times \operatorname{age}_{i}^{2}+\eta_{i 2}(s),
\end{aligned}
$$

where $\lambda_{i, k}$ are the three eigenvalues the $i$-th subject for $k=1,2,3$, and $g_{i}$ and age $_{i}$ denote gender and age, respectively. In this case, $m=2, B(s)=\left(\beta_{j k}(s)\right)$ is a $2 \times 4$ matrix, and $\mathbf{x}_{i}=\left(1, \mathrm{~g}_{i}, \text { age }_{i}, \operatorname{age}_{i}^{2}\right)^{T}$. It is trivial to extend model (2) to other nonlinear and nonparametric functions of age [13.

To estimate the coefficient functions in $B(s)$, we develop an adaptive local polynomial kernel smoothing technique 14, whereas it is possible to use spline type of methods including B-spline and smoothing spline. Specifically, using Taylor's expansion, we can expand $B_{k}\left(s_{j}\right)$ at $s$ to obtain $B_{k}\left(s_{j}\right)=B_{k}(s)+\dot{B}_{k}(s)\left(s_{j}-s\right)=$ $A_{k}(s) \mathbf{z}_{h_{n_{G}, k}}\left(s_{j}-s\right)$, where $\mathbf{z}_{h_{n_{G}, k}}\left(s_{j}-s\right)=\left(1,\left(s_{j}-s\right) / h_{n_{G}, k}\right)^{T}$ and $A_{k}(s)=$ $\left[B_{k}(s), h_{n_{G}, k} \dot{B}_{k}(s)\right]$ is a $p \times 2$ matrix, in which $\dot{B}_{k}(s)=\left(\dot{b}_{k 1}(s), \cdots, \dot{b}_{k p}(s)\right)$ is a $p \times 1$ vector and $\dot{b}_{k l}(s)=d b_{k l}(s) / d s$ for $l=1, \cdots, p$. We calculate a weighted least squares estimate of $A_{k}(s)$ as follows. Let $K(\cdot)$ be a kernel function, such as the Gaussian and uniform kernels [14]. For a fixed bandwidth $h$ and each $k$, we estimate $A_{k}(s)$ by minimizing an objective function given by

$$
\sum_{i=1}^{n} \sum_{j=1}^{n_{G}}\left[y_{i, k}\left(s_{j}\right)-\mathbf{x}_{i}^{T} A_{k}(s) \mathbf{z}_{h_{n_{G}, k}}\left(s_{j}-s\right)\right]^{2} K_{h_{n_{G}, k}}\left(s_{j}-s\right),
$$

where $K_{h_{n_{G}, k}}(\cdot)=K\left(\cdot / h_{n_{G}, k}\right) / h_{n_{G}, k}$ is a rescaled kernel function. For each $k$, we pool the data from all $n$ subjects and select an optimal bandwidth $h_{n_{G}, k}$, denoted by $\hat{h}_{k, o}^{(1)}$, by minimizing the cross-validation score. Based on $\hat{h}_{k, o}^{(1)}$, we can obtain an estimate of $B_{k}(s)$, denoted by $\hat{B}_{k, o}(s)$.

\subsection{Smoothing Individual Functions and Covariance Estimating}

To simultaneously construct all individual functions $\eta_{i, k}(s)$, we also employ the local polynomial kernel smoothing technique [14. Specifically, using Taylor's expansion, we can expand $\eta_{i, k}\left(s_{j}\right)$ at $s$ to obtain $\eta_{i, k}\left(s_{j}\right)=\mathbf{d}_{i, k}(s)^{T} \mathbf{z}_{h_{n_{G}, k}^{(2)}}\left(s_{j}-s\right)$, where $\mathbf{d}_{i, k}(s)=\left(\eta_{i, k}(s), h_{k}^{(2)} \dot{\eta}_{i, k}(s)\right)^{T}$ is a $2 \times 1$ vector. We develop an algorithm to estimate $\mathbf{d}_{i, k}(s)$ as follows. For each $k$ and a fixed bandwidth $h_{n_{G}, k}^{(2)}$, we estimate $\mathbf{d}_{i, k}(s)$ by minimizing an objective function given by

$$
\sum_{j=1}^{n_{G}}\left[y_{i, k}\left(s_{j}\right)-\mathbf{x}_{i}^{T} \hat{B}_{k, o}\left(s_{j}\right)-\mathbf{d}_{i, k}(s)^{T} \mathbf{z}_{h_{n_{G}, k}^{(2)}}\left(s_{j}-s\right)\right]^{2} K_{h_{n_{G}, k}^{(2)}}\left(s_{j}-s\right) .
$$

For each $k$, we pool the data from all $n$ subjects and select the optimal bandwidth $h_{n_{G}, k}$, denoted by $\hat{h}_{k, o}^{(2)}$, by minimizing the generalized cross-validation score. 
Based on $\hat{h}_{k, o}^{(2)}$, we can estimate $\eta_{i, k}(s)$ and $\boldsymbol{\eta}_{i}(s)$, denoted by $\hat{\eta}_{i, k o}(s)$ and $\hat{\boldsymbol{\eta}}_{i, o}(s)$, respectively, for all $i$ and $k$.

After obtaining $\hat{\boldsymbol{\eta}}_{i, o}(s)$, we can estimate the mean function $\boldsymbol{\eta}(s)$ and the covariance function $\Sigma_{\eta}(s, t)$ of $\boldsymbol{\eta}_{i}(s)$. Specifically, we estimate $\boldsymbol{\eta}(s)$ and $\Sigma_{\eta}(s, t)$ by using their empirical counterparts of the estimated $\hat{\boldsymbol{\eta}}_{i, o}(s)$ as follows: $\hat{\boldsymbol{\eta}}_{o}(s)=$ $n^{-1} \sum_{i=1}^{n} \hat{\boldsymbol{\eta}}_{i, o}(s)$ and $\hat{\Sigma}_{\eta}(s, t)=(n-m)^{-1} \sum_{i=1}^{n} \hat{\boldsymbol{\eta}}_{i, o}(s) \hat{\boldsymbol{\eta}}_{i, o}(t)^{T}$.

We construct a nonparametric estimator of the covariance matrix $\Sigma_{\epsilon}(s, s)$ as follows. Let $\hat{B}_{o}(s)=\left[\hat{B}_{1, o}(s), \cdots, \hat{B}_{m, o}(s)\right]$ and $\hat{\boldsymbol{\epsilon}}_{i}\left(s_{j}\right)=\mathbf{y}_{i}\left(s_{j}\right)-\hat{B}_{o}\left(s_{j}\right)^{T} \mathbf{x}_{i}-$ $\hat{\boldsymbol{\eta}}_{i, o}\left(s_{j}\right)$ be estimated residuals for $i=1, \cdots, n$ and $j=1, \cdots, n_{G}$. We consider an estimate of $\Sigma_{\epsilon}(s, s)$ given by $\hat{\Sigma}_{\epsilon}(s, s)=(n-m)^{-1} \sum_{i=1}^{n} \sum_{j=1}^{n_{G}} \tilde{K}_{h^{(3)}}\left(s_{j}-\right.$ $s)\left[\hat{\boldsymbol{\epsilon}}_{i}\left(s_{j}\right)\right]^{\otimes 2}$, where $\tilde{K}_{h^{(3)}}\left(s_{j}-s\right)=K_{h^{(3)}}\left(s_{j}-s\right) / \sum_{j=1}^{n_{G}} K_{h^{(3)}}\left(s_{j}-s\right)$. To select the optimal bandwidth $h^{(3)}$, denoted by $\hat{h}_{o}^{(3)}$, we minimize the cross-validation score. Based on $\hat{h}_{o}^{(3)}$, we can estimate $\Sigma_{\epsilon}(s, s)$, denoted by $\hat{\Sigma}_{\epsilon o}(s, s)$.

\subsection{Test Statistics and Resampling Method}

In neuroimaging studies, most scientific questions require the comparison of fiber bundle diffusion properties along fiber bundles across two (or more) diagnostic groups and the assessment of the development of fiber bundle diffusion properties along time. Such questions can often be formulated as linear hypotheses of $B(s)$ as follows: $H_{0}: \mathbf{C v e c}(B(s))=\mathbf{b}_{0}(s)$ for all $s$ vs. $H_{1}: \mathbf{C v e c}(B(s)) \neq \mathbf{b}_{0}(s)$, where $\mathbf{C}$ is a $r \times m p$ matrix of full row rank and $\mathbf{b}_{0}(s)$ is a given $r \times 1$ vector of functions. We propose both local and global test statistics. The local test statistic can identify the exact location of significant grid point on a specific tract. At a given grid point $s_{j}$ on a specific tract, we test the local null hypothesis $H_{0}\left(s_{j}\right): \mathbf{C v e c}\left(B\left(s_{j}\right)\right)=\mathbf{b}_{0}\left(s_{j}\right)$ using a local test statistic $S_{n}\left(s_{j}\right)=n \mathbf{d}\left(s_{j}\right)^{T}\left[\mathbf{C}\left(\hat{\Sigma}_{\eta}\left(s_{j}, s_{j}\right) \otimes \hat{\Omega}_{X}^{-1}\right) \mathbf{C}^{T}\right]^{-1} \mathbf{d}\left(s_{j}\right)$, where $\hat{\Omega}_{X}=n^{-1} \sum_{i=1}^{n} \mathbf{x}_{i}^{\otimes 2}$ and $\mathbf{d}(s)=\mathbf{C v e c}\left(\hat{B}_{o}(s)-\mathbf{b}_{0}(s)\right)$. We test the null hypothesis $H_{0}: \operatorname{Cvec}(B(s))=\mathbf{b}_{0}(s)$ for all $s$ using a global test statistic $S_{n}=$ $n \int_{0}^{L_{0}} \mathbf{d}(s)^{T}\left[\mathbf{C}\left(\hat{\Sigma}_{\eta}(s, s) \otimes \hat{\Omega}_{X}^{-1}\right) \mathbf{C}^{T}\right]^{-1} \mathbf{d}(s) d s$, where $L_{0}$ is the whole arc length of a specific fiber bundle. In order to use $S_{n}$ as a test statistic, we can show that $S_{n}$ has appropriate asymptotic distribution as $n \rightarrow \infty$. We develop a resampling method (or wild bootstrap method) to approximate the $p$-value of $S_{n}$. The key ideas are to fit model (11) under the null hypothesis $H_{0}$, which yields $\hat{B}_{o}^{*}\left(s_{j}\right), \hat{\boldsymbol{\eta}}_{i, o}^{*}\left(s_{j}\right)$ and $\hat{\boldsymbol{\epsilon}}_{i, o}^{*}\left(s_{j}\right)$ for $i=1, \cdots, n$ and $j=1, \cdots, n_{G}$, and then to generate random samples from the fitted model in order to approximate the null distribution of $S_{n}$.

\section{Results}

Clinical Data. Twenty four healthy rhesus monkeys (male and female included) at the Harlow Primate Laboratory with age between 10 to 72 months were scanned on a 3 Tesla GE scanner (SIGNA Excite) with a high-resolution 3DSPGR sequence $\left(0.2344 \times 0.2344 \times 0.4980 \mathrm{~mm}^{3}\right)$, a T2-weighted spin-echo sequence $\left(0.2344 \times 0.2344 \times 1.5 \mathrm{~mm}^{3}\right)$ and a 12 -direction diffusion-weighted EPI sequence $\left(0.5469 \times 0.5469 \times 2.5 \mathrm{~mm}^{3}\right)$. After DTI estimation, a nonlinear fluid deformation based high-dimensional, unbiased atlas computation method was used 
(a)

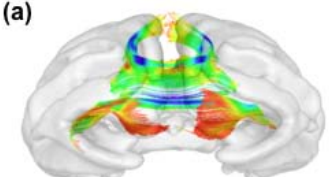

(b)

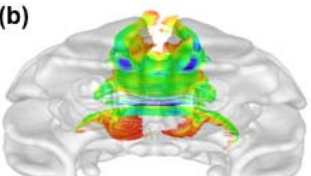

(c)

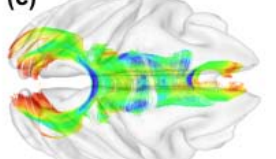

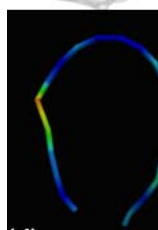

(d)

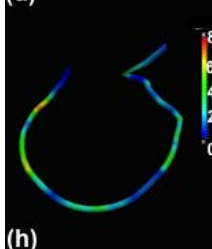

(e)
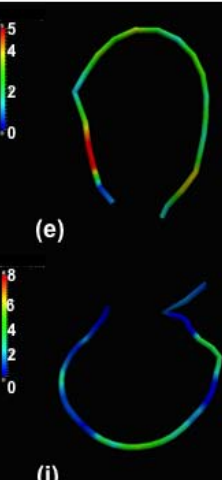

(i)

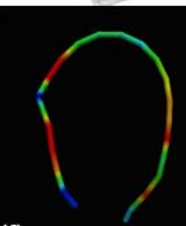

(f)

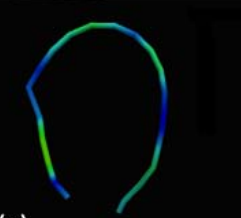

(g)

Fig. 2. Results from a study of neurodevelopment in healthy rhesus monkeys: panels (a)-(c): (a) anterior, (b) posterior, and (c) superior views of corpus callosum tracts; panels (d)-(g): $-\log _{10}(p)$-values of $S_{n}\left(s_{j}\right)$ for testing time effect in the genu tract: (d) $\lambda_{1}$, (e) $\lambda_{(2,3)}$, (f) FA, (g) MD; panels (h)-(k): $-\log _{10}(p)$-values of $S_{n}\left(s_{j}\right)$ for testing time effect in the splenium tract: (d) $\lambda_{1}$, (e) $\lambda_{(2,3)}$, (f) FA, (g) MD

to carry out a large deformation non-linear registration [15]. Detailed information regarding the DTI atlas building procedure has been described in [9]. Major fiber bundles are tracked in the atlas space within 3D Slicer (www.slicer.org). With the fiber bundles in atlas space, each subject's DTI data is transformed into the atlas space. For each subject at a given time point, the data within the fiber bundle is parameterized as a sampled function of equidistance steps along the fiber. The result of the procedure is thus a set of corresponding sampled functions, including FA, MD, etc. parameterized by arc length from the atlas fiber tract for each individual subject using invert of the atlas-building transformation. These sampled functions at each point along the fiber tract were then used to study the effect of age, gender and other covariates on neural development.

For the sake of space, we chose two tracts of interest including the splenium and genu of the corpus callosum tract and then computed fractional anistropy (FA), mean diffusivity (MD), and $\lambda_{1}$ and $\lambda_{(2,3)}$ of diffusion tensors at each grid point on both tracts for each of the 24 monkeys. FA denotes the inhomogeneous extent of local barriers to water diffusion, while MD measures the averaged magnitude of local water diffusion. The three eigenvalues of diffusion tensor may, respectively, reflect the magnitude of water diffusivity along and perpendicular to the long axis of white matter fibers [16].

We applied VCMTS to the joint analysis of $\lambda_{1}, \lambda_{(2,3)}, \mathrm{FA}$, and MD values along the splenium tract as follows. We fitted the functional linear model (2) to these 
four diffusion properties from all 24 subjects, in which $\mathbf{x}_{i}=\left(1, \mathrm{~g}_{i}, \text { age }_{i}, \text { age }_{i}^{2}\right)^{T}$ and $m=2$, and then we estimated the function of regression coefficient vector $\hat{B}(s)$. Secondly, we constructed the global test statistic $S_{n}$ to test the effects of all the age effect for each of the four diffusion properties, and performed hypothesis testing on the whole splenium and genu tracts. The $p$-value of $S_{n}$ was approximated using the resampling method with $G=10,000$.

We considered the genu tract and performed hypothesis testing on time effect for the whole tract. The $p$-values of $S_{n}$ corresponding to $\lambda_{1}, \lambda_{(2,3)}, \mathrm{FA}$, and MD equal $0.31,0.19,0.007$, and 0.29 , respectively. This indicates a significant change of the degrees of anisotropy, not the degree of diffusivity, along the genu tract. We further performed hypothesis testing at each grid point along the splenium tract (Figs. 2). For $\lambda_{1}, \lambda_{(2,3)}$, and MD, no significant effect of time effect was found, even though the $-\log _{10}(p)$ values of $S_{n}(s)$ for age at several single grid points were slightly greater than 2 (Fig. 2 (d), (e), (g)). For FA alone, the effects of time were significant in the middle and tail of the genu tract (Fig. 2 (f)).

We considered the splenium tract and performed hypothesis testing on time effect for the whole tract. The $p$-values of $S_{n}$ corresponding to $\lambda_{1}, \lambda_{(2,3)}, \mathrm{FA}$, and MD equal 0.001, 0.002, 0.000, and 0.004, respectively. This indicates a significant change of the degrees of diffusivity and anisotropy, along the splenium tract. We further performed hypothesis testing at each grid point along the splenium tract (Fig. $2(\mathrm{~h})-(\mathrm{k})$ ). For all diffusion properties, the effects of time were significant in most of grid points along the splenium tract (Fig. $2(\mathrm{~h})-(\mathrm{k})$ ).

\section{Discussion}

We have developed VCMTS for statistically analyzing multiple diffusion properties along fiber bundle and assessing their association with a set of covariates in real applications. The proposed methodology is demonstrated in a study of neurodevelopment in rhesus monkey. Significant time effect on multiple diffusion properties were examined and localized in two representative tracts. VCMTS is able to delineate the complex inhomogeneous spatial-temporal maturation patterns as the apparent changes in FA, MD, and the eigenvalues of diffusion tensors. Specifically, our results suggest that white matter maturation patterns are different in different white matter regions. We expect that this novel statistical tool will lead to new findings in our clinical applications.

\section{References}

1. Smith, S.M., Jenkinson, M., Johansen-Berg, H., Rueckert, D., Nichols, T.E., Mackay, C.E., Watkins, K.E., Ciccarelli, O., Cader, M., Matthews, P., Behrens, T.E.: Tractbased spatial statistics: voxelwise analysis of multi-subject diffusion data. NeuroImage 31, 1487-1505 (2006)

2. O'Donell, L.J., Westin, C.F., Golby, A.J.: Tract-based morphometry for white matter group analysis. NeuroImage 45, 832-844 (2009)

3. Snook, L., Plewes, C., Beaulieu, C.: Voxel based versus region of interest analysis in diffusion tensor imaging of neurodevelopment. NeuroImage 34, 243-252 (2007) 
4. Snook, L., Paulson, L.A., Roy, D., Phillips, L., Beaulieu, C.: Diffusion tensor imaging of neurodevelopment in children and young adults. NeuroImage 26, 1164-1173 (2005)

5. Worsley, K.J., Taylor, J.E., Tomaiuolo, F., Lerch, J.: Unified univariate and multivariate random field theory. Neuroimage 23, 189-195 (2004)

6. Hecke, W.V., Sijbers, J., Backer, S.D., Poot, D., Parizel, P.M., Leemans, A.: On the construction of a ground truth framework for evaluating voxel-based diffusion tensor mri analysis methods. NeuroImage 46, 692-707 (2009)

7. Jones, D.K., Symms, M.R., Cercignani, M., Howard, R.J.: The effect of filter size on vbm analyses of dt-mri data. NeuroImage 26, 546-554 (2005)

8. Yushkevich, P.A., Zhang, H., Simon, T., Gee, J.C.: Structure-specific statistical mapping ofwhite matter tracts. Neuroimage 41, 448-461 (2008)

9. Goodlett, C.B., Fletcher, P.T., Gilmore, J.H., Gerig, G.: Group analysis of dti fiber tract statistics with application to neurodevelopment. NeuroImage 45, S133-S142 (2009)

10. Gouttard, S., Prastawa, M., Bullitt, E., Lin, W.L., Goodlett, C., Gerig, G.: Constrained data decomposition and regression for analyzing healthy aging from fiber tract diffusion properties. In: Yang, G.-Z., Hawkes, D., Rueckert, D., Noble, A., Taylor, C. (eds.) MICCAI 2009. LNCS, vol. 5761, pp. 321-328. Springer, Heidelberg (2009)

11. Zhu, H.T., Styner, M., Tang, N.S., Liu, Z.X., Lin, W.L., Gilmore, J.: Frats: Functional regression analysis of dti tract statistics. IEEE Transactions on Medical Imaging 29, 1039-1049 (2010)

12. Zhu, H.T., Li, R.Z., Kong, L.N., Styner, M., Gilmore, J.: Multivariate varying coefficient models with applications for dti tract statistics. Technical report, University of the North Carolina (2010)

13. Fan, J., Zhang, W.: Statistical methods with varying coefficient models. Statistics and Its Interface 2 (2008)

14. Fan, J., Gijbels, I.: Local Polynomial Modelling and Its Applications. Chapman and Hall, London (1996)

15. Joshi, S., Davis, B., Jomier, M., Gerig, G.: Unbiased diffeomorphic atlas construction for computational anatomy. Neuroimage 23, S151-S160 (2004)

16. Song, S.K., Sun, S.W., Ju, W.K., Lin, S.J., Cross, A.H., Neufeld, A.H.: Diffusion tensor imaging detects and differentiates axon and myelin degeneration in mouse optic nerve after retinal ischemia. Neuroimage 20, 1714-1722 (2003) 